THE KURUME MEDICAL JOURNAL

Vol. 41, p. $81-85,1994$

\title{
Clinical Analysis of Nineteen Patients with Traumatic Subdural Hygromas
}

\author{
AKIRA ISHIBASHI, YOSHITAKE YOKOKURA* AND JUN MIYAGI** \\ Departments of Neurosurgery and Surgery* ${ }^{*}$ Yokokura Hospital, 394 Takada-machi, \\ Miike-gun, 839-02 and ${ }^{* *}$ Department of Neurosurgery, Kurume University School of \\ Medicine, Kurume, 830 Japan
}

Received for publication March 22, 1994

\begin{abstract}
Summary: Nineteen patients with traumatic subdural hygroma (TSH) who were admitted between 1988 and 1992, were reviewed. Diagnosis of TSH was made by serial computerized tomography (CT) after initial head injury, and patients were followed for up to 19 months after head injury. The patients ranged in age from 53 to 91 years (mean 75.6 yrs). Fifteen patients were treated conservatively, and 4 patients underwent surgery. Patients had disturbance of consciousness (transient in 5 cases, persisting in 3 cases), headache (10 cases), vomiting ( 6 cases), and vertigo ( 2 cases). TSH began to develop within 24 hs in 10 patients after initial head injury, and in $13(68 \%)$ of 19 patients, TSH was demonstrated within 72 hs after the initial head injury. Chronic subdural hematoma (CSH) developed in 5 (26\%) of 19 patients and 2 patients underwent surgery. In 15 patients, who were treated conservatively, TSH disappeared in 10 patients, and decreased in 4 patients, but remained unchanged in 1 patient. Clinically, 11 patients improved. Three patients remained unchanged. One patient, who had cerebral contusion, died of pneumonia. In 4 patients, who underwent surgery, including 2 patients with CSH, TSH disappeared in all patients. Two patients improved, and 1 patient was unchanged. Another patient, who had cerebral contusion and normal pressure hydrocephalus, died. In general, clinical outcome was satisfactory, except for the patients who had accompanying parenchymal lesions before or after head injury. Surgical treatment was limited to the patients who had rapidly enlarging TSH and neurological deterioration.
\end{abstract}

Key words: subdural hygroma - computed tomography — head injury subdural effusion - cerebral contusion

\section{Introduction}

With the advent of neuro-imaging techniques, such as computerized tomography (CT), the determination of the presence and the extent of the intracranial abnormal pathology has been significantly facilitated. Recently, the authors have diagnosed 19 patients with traumatic subdural hygroma (TSH), which was identified by serial CT. Various theories have been proposed about the mechanism contributing to the development of TSH after head injury (Nattziger, 1924; Wetterling et al. 1988), but its pathogenesis still remains unclear. 
In this article, the authors report the results of the management of these 19 patients with TSH, and discuss the pathogenesis as well as the surgical treatment of TSH.

\section{Materials and Methods}

The medical records of 19 patients with TSH, who were admitted to our institution between 1988 and 1992, were reviewed. The patients ranged in age from 53 to 91 years (mean 75.6 yrs). Sixteen were males and 3 were females (Table 1). On admission, 8 patients suffered from disturbances of consciousness. Subsequently, 5 patients recovered their consciousness within a few hours, while 3 patients remained in unconscious state (one in I-2, and two in II-30/Japan coma scale). Ten patients complained of headache. Five patients had vertigo and 2 patients had vomiting

TABLE 1.

\begin{tabular}{lc}
\multicolumn{2}{c}{ Summary of nineteen patients } \\
\hline Male & 16 \\
Female & 3 \\
Mean Age & $75.6 \mathrm{yrs}$ \\
& $(53 \mathrm{yrs}-91 \mathrm{yrs})$
\end{tabular}

TABLE 2.

Clinical symptoms of nineteen patients on admission

\begin{tabular}{lc} 
& cases \\
\hline Disturbance of consciousness & 8 \\
Headache & 10 \\
Vertigo & 6 \\
Vomiting & 2 \\
\hline
\end{tabular}

(Table 2). Nineteen patients were followed up and examined serially by CT after head injury. Evaluation by CT was done for up to 19 months following the initial head injury.

\section{Results}

As to the site of TSH, 15 patients showed bilateral TSH and 4 patients had unilateral TSH (right side in two, and left in two). The accompanying intracranial unusual lesions were as follows: cerebral contusion (two), cerebral infarction (one), normal pressure hydrocephalus (NPH, one), traumatic subarachnoid hemorrhage (one), and acute subdural hematoma (one) (Table 3).

TSH began to develop within 24 hs after the initial head injury in 10 patients. In particular, frequent CT scans disclosed the appearance of TSH

TABLE 3.

Associated intracranial lesions

\begin{tabular}{ll}
\hline Cerebral contusion & 2 \\
Cerebral infarction & 1 \\
Normal pressure hydrocephalus & 1 \\
Acute subdural hematoma & 1 \\
Subarachnoid hemorrhage & 1 \\
\hline
\end{tabular}

TABLE 4.

The accumulation of TSH identified by serial $C T$

\begin{tabular}{rc}
\hline \multicolumn{1}{c}{ hours } & cases \\
\hline$<24$ & 10 \\
$24-48$ & 2 \\
$48-72$ & 1 \\
$>72$ & 6 \\
\hline
\end{tabular}


within $1 \mathrm{~h}$ after head injury in 3 of these 10 patients (Table 4). This fact implies that the accumulation of subdural fluid occurred in the early stages of head injury. Five (26\%) of 19 patients subsequently developed chronic subdural hematoma (CSH), which was revealed by CT scan between Day 13th and Day 145th (mean: Day 67.2). Four patients underwent surgery. In 2 of these 4 patients, irrigation of subdural hygroma was performed on Day 5 and on Day 15 following head injury. In both patients, when the dura mater was excised, no formation of neomembrane was found. In one of the 2 patients who later developed NPH, a V-P shunting operation was done. In other 2 patients, who were operated on Day 33 and on Day 95, surgery demonstrated CSH with neomembrane under the dura mater. The remaining 15 patients were treated conservatively.

Table 5 shows the clinical results of

TABLE 5.

Clinical outcome of nineteen patients

\begin{tabular}{lcc}
\hline & $\begin{array}{c}\text { Conservative } \\
\text { treatment (15) }\end{array}$ & operation (4) \\
\hline Improved & 11 & 2 \\
Unchanged & 3 & 1 \\
Dead & 1 & 1 \\
\hline
\end{tabular}

TABLE 6.

Follow-up evaluation of TSH by serial CT

\begin{tabular}{lcc}
\hline & $\begin{array}{c}\text { Conservative } \\
\text { treatment (15) }\end{array}$ & operation (4) \\
\hline Disappeared & 10 & 4 \\
Decreased & 4 & 0 \\
Unchanged & 1 & 0 \\
\hline
\end{tabular}

19 patients with TSH. Of the 4 patients who underwent surgery, 2 became free from headache and/or vertigo soon after surgery. In 1 patient, who was demented before head injury, the symptoms remained unchanged. One patient died of pneumonia, under the persisting disturbed consciousness due to cerebral contusion, accompanied by NPH. In the 14 patients who were treated conservatively, symptoms improved in 11 and were unchanged in 3 patients. One patient died of pneumonia with persisting impaired consciousness due to cerebral contusion.

Serial CT scans disclosed that in all 4 patients with TSH, (including 2 patients with $\mathrm{CSH}$ ), who were operated on, TSH disappeared between Day 30th and Day 180th (mean: Day 99) after head injury (Table 6). In the remaining 15 patients who were treated without surgical intervention, TSH disappeared in 10 patients between Day 14th and Day 284th (mean: Day 76), and decreased in 4 patients. TSH remained unchanged in 1 patient. Thus, in 14 (93\%) of 15 patients who were treated conservatively, TSH disappeared or decreased. It was also noted that the accumulation of TSH began between $1 \mathrm{~h}$ and $72 \mathrm{hs}$, and the evolution of TSH was identified between 1 day and 24 days. Furthermore, the resolution of TSH began between 6 days and 36 days (mean 21 days).

\section{Discussion}

With the development of neuroimaging techniques such as CT which has become routinely available in most institutions, the diagnosis of $\mathrm{TSH}$ has 
been made easier in a patient with head injury. The use of serial investigations by CT after the initial head injury has been an especially important and useful method for detecting TSH when TSH was not found with an initial CT. Therefore, a recent report described a higher incidence of TSH (21.6\%) (Murata, 1993) than reported in earlier studies (0.8 6.6\%) (French et al. 1978; Tanikake et al. 1979). As to the age distribution in patients with TSH, elderly patients were prominent (mean 75.6 yrs) in the present study.

The impact of the trauma to the head did not seem as severe as had been described (Kaneko et al. 1988), because clinical symptoms in most patients with TSH were mild. The pathogenesis of TSH is still unclear, and various factors contributing to the development of TSH are described. (Nattziger, 1924; Da Costa and Adson, 1941; Miura et al. 1975; French et al. 1978; Fujioka et al. 1981; Stone et al.1981; Yoshimasu et al. 1981; Wetterling et al. 1988).

The present study suggested that there might be a different mechanism between the initial accumulation of TSH in the early stage and the evolution and resolution of TSH in the chronic stage.

In this study, the initial accumulation of TSH was found to develop between $1 \mathrm{~h}$ and $72 \mathrm{hs}$ in $13(68 \%)$ of 19 patients after the initial head injury. Furthermore, the evolution of TSH began between 1 day and 24 days after the initial head injury, leading to the beginning of the resolution of TSH between 6 and 36 days after the initial head injury. In 14 of 15 patients (93\%), who were treated conservatively, TSH disappeared or decreased within almost 9 months. From the above clinical observations, it is conceivable that the arachnoid tear might be the most important factor for the rapid accumulation of TSH in the early stage, though there are only three reports describing the arachnoid tear which was noted during the surgical procedure (Da Costa and Adson, 1941; Miura et al. 1975; Stone et al. 1981).

In regard to the mechanism of the evolution and resolution of TSH in the chronic stage, other different pathophysiologies should be considered, secondary to the arachnoid tear that occurred after the initial head injury in the early stage.

In the present study, 2 patients with TSH were operated on in the early stage (on Day 5 and on Day 15), but no new membrane formations under the dura mater were identified during surgery. In contrast to our operative finding, neomembrane formations under the dura mater were noted during surgery in the chronic stage, and it was suggested that inflow and outflow of fluid might occur through neovascularized membrane under the dura mater (Oka et al. 1972; Hasegawa et al. 1992). Thus far, it has been suggested that this neovascularized membrane might be closely associated with the evolution and resolution of TSH, in the chronic stage, but further investigations are needed for an accurate determination of the pathogenesis of the evolution and resolution of TSH in the chronic stage.

Consequently, TSH disappeared or decreased within almost 9 months after the initial head injury in most patients who were observed without surgical treatment. Spontaneous resolution of TSH was expected, and the clinical course 
was generally satistactory, except for the patients who had an accompanying parenchymal lesions before or after head injury. So the surgical treatments should be reserved for the patients, who have TSH with rapid expansion and an apparent mass effect contributing to neurological deterioration.

\section{References}

Da Costa, D.G. and Adson, A.W. (1941). Subdural hygroma. Arch. Surg. 43, 559-567.

French, N.B., Cobb, C.A., III, Corkill, G. and Youmans, J.R. (1978). Delayed evolution of post-traumatic subdural hygroma. Surg. Neuro. 9, 145-148.

Fujioka, S., Matsuokado, Y., Kaku, M., Sakurama, N., Nonaka, N. et al. (1981). CT analysis of 100 cases with chronic subdural hematoma with regard to clinical manifestation and the enlarging process of the hematoma. Neurol. Med. Clin. (Tokyo) 21, 1153-1160. (in Japanese)

Hasegawa, M., Yamashita, T., Yamashita, J., Suzuki, M. and Shimada, S. (1992). Traumatic subdural hygroma: pathology and meningeal enhancements on magnetic resonance imaging. Neurosurgery 31, 580-585.

Kaneko, T., Hirai, O., Nishikawa, M., Munaka, M., Watanabe, O. et al. (1988). Analysis of 24 cases of subdural fluid collections.
Neurotraumatology 11, 73-77.

Miura, F., Fuchiune, T., Machiyama, Y., Satoh, K., Shirata, N. et al. (1975). Acute subdural hygroma in the posterior fossa. No Shinkei Geka 3, 769-776. (in Japanese)

Murata, K. (1993). Chronic subdural hematoma may be preceded by persistent traumatic subdural effusion. Neurol. Med. Clin. 33, 691-696. (in Japanese)

NatTZiger, H.C. (1924). Subdural fluid accumulations following head injury. JAMA $\mathbf{8 2}$, 1751-1752.

Oка, Н., Мотомасні, Y., Suzuki, Y. and Ando, K. (1972). Subdural hygroma after head injury. Acta Neurochir. 26, 265-273.

Stone, J.L., Lang, R.G.R., Sugar, O. and Moody, R.A. (1981). Traumatic subdural hygroma. Neurosurgery 8, 542-550.

Tanikake, T., Kawamura, K., Takemura, K., Kinugawa, K., Karasawa, J. et al. (1979). Diagnosis and follow up studies of traumatic subdural fluid collection. CT Kenkyu 1, 385-392. (in Japanese)

Wetterling, T., Demierre, B., Roma, B. and Nekic, M. (1988). Protein analysis of subdural hygroma fluid. Acta Neurochir. (Wien) 91, 79-82.

Yoshimasu, N., Tamura, A., WaKai, $\mathrm{S}$. and Yosнiмото, S. (1981). Evolution from acute subdural hemorrhage to chronic subdural hematoma: Observation of subdural hematoma by computerized tomography. No Shinkei Gaka 9, 1025-1031. (in Japanese) 